\title{
Mycoplasmas in rheumatoid arthritis and other human arthritides
}

The possibility that mycoplasmas might be a cause of human arthritis has been considered from time to time over almost 60 years and has again become a topic of interest. Mycoplasmas are small, ubiquitous bacteria without a rigid cell wall existing as commensals and pathogens of both animals and humans. Their small genome size, varying from 580 to 2200 kilobases, is comparable with that of large viruses and results in limited synthetic capability. Thus, they are dependent on nutrients from eukaryotic cells for growth, and are often difficult to isolate and culture. More than one type of mycoplasma has been incriminated as a cause of arthritis in most animal species. In some instances-for example, the Mycoplasma arthritidis induced arthritis of rats, the condition is acute and purulent. In other cases, however, a chronic or relapsing polyarthritis develops. Chronic, severe and destructive polyarthritides due, for instance, to $M$ hyosynoviae or $M$ hyorhinis in swine, and to $M$ capricolum in goats and sheep, have often been compared with human rheumatoid arthritis. Indeed, they are characterised by a massive proliferation of the synovial membrane, with infiltration of neutrophils, lymphocytes and macrophages, which produces bone and cartilage erosion, and may persist for the life of the animal. Many experimental models of mycoplasma induced arthritis have been developed. Interestingly, in some of these, although arthritis has persisted, the mycoplasmas have been recoverable from the joint tissues only in the early stage of disease. ${ }^{1}$

In humans, mycoplasmas have been implicated in septic arthritis occurring in different immunodeficiency conditions, particularly in patients with hypogammaglobulinaemia. ${ }^{2}$ However, the finding of mycoplasmas in animal arthritides, and the anti-mycoplasmal activity of gold salts and their beneficial effect in rheumatoid arthritis, provided the impetus originally to search for mycoplasmas in the joints of patients with rheumatoid arthritis. Thus, 30 years ago there were reports of the isolation of mycoplasmas, not viruses, from rheumatoid synovial samples that had been passed through cell cultures before being introduced into mycoplasmal medium. ${ }^{1}$ It became apparent, however, that the mycoplasmas did not come from the affected joints but from the cell cultures which were apparently contaminated by these organisms and created a false impression. No more convincing were the occasional and scattered reports of the isolation of mycoplasmas from rheumatoid joints by direct inoculation of mycoplasmal media without recourse to the use of cell cultures. Indeed, most of the earlier reports were unsubstantiated by identification of the isolated organisms and a specific mycoplasma was never isolated consistently from rheumatoid synovial tissues by workers in different laboratories. However, in the late 1960 s the isolation of $M$ fermentans from the joints of about two fifths of patients with rheumatoid arthritis and from one fifth of those without rheumatoid arthritis was reported. ${ }^{3}$ The use of a sucrose density gradient was claimed to be the key to success. By this technique, organisms were considered to be concentrated and separated from synovial fluid inhibitors and the bands corresponding to the density of mycoplasmas were then introduced into conventional mycoplasmal medium. However, those who used the same method subsequently failed to substantiate the findings. ${ }^{1}$ Although a rare isolate of $M$ fermentans has come from efforts in which the centrifugation technique has not been used, even recently, ${ }^{4}$ the general failure has been such that the idea of this mycoplasma as a serious contender for an aetiological role in rheumatoid arthritis or other arthritides has fallen by the wayside. ${ }^{5}$ More recently, the application of the polymerase chain reaction (PCR) technique to the detection of $M$ fermentans has resulted in it being found in the oropharynx of about $20 \%$ of subjects attending sexually transmitted disease clinics and also in the blood of $10 \%$ and the urine of $5 \%$ of these subjects. ${ }^{6}$ Such common occurrence and the superior sensitivity of the PCR assay as a means of detection have stimulated renewed efforts to seek $M$ fermentans in rheumatoid arthritis and other arthritides. In recent years there have been reports of the association of $M$ pneumoniae and $M$ genitalium with arthropathy. Thus, $M$ pneumoniae has been associated with arthropathy in children and young adults. ${ }^{7}$ Interestingly, in Canada concordance has been shown between the annual incidence of $M$ pneumoniae infections in children and the incidence of juvenile rheumatoid arthritis. In contrast, there was no correlation between juvenile rheumatoid arthritis and any viral infection. ${ }^{8}$ In addition, $M$ pneumoniae together with $M$ genitalium has been detected in the synovial fluid of a patient with pneumonia and polyarthritis. ${ }^{9}$ Furthermore, $M$ genitalium has been detected by PCR in the joints of two of 13 patients with arthritis, one with sexually acquired reactive arthritis and the other with seronegative rheumatoid arthritis. ${ }^{10}$ It is clear, therefore, that the issue of the role of mycoplasmas in inflammatory rheumatic disorders of unknown cause, including rheumatoid arthritis, can no longer be ignored. Several mycoplasmal species are detectable in the joints. Whether $M$ fermentans is a major contributor and whether the presence of mycoplasmas is a consequence rather than a cause of the arthritis remains to be seen.

D TAYLOR-ROBINSON

MRC Sexually Transmitted Diseases Research Group,

fefferiss Trust Laboratories,

Imperial College School of Medicine at St Mary's, Paddington,

London W2 $1 N Y$

T SCHAEVERBEKE

Service de Rheumatologie,

Hôpital Pellegrin,

Centre Hospitalier Universitaire de Bordeaux,

Bordeaux, France

1 Taylor-Robinson D, Taylor G. Do mycoplasmas cause rheumatic disease? n: Dumonde DC, ed. Infection and immunology in the rheumatic diseases. Oxford: Blackwell Scientific Publications, 1976:177-86.

2 Furr PM, Taylor-Robinson D, Webster ADB. Mycoplasmas and ureaplasmas in patients with hypogammaglobulinaemia and their role in arthritis: microbiological observations over twenty years. Ann Rheum Dis 1994;53: 183-7. 
3 Williams MH. Recovery of mycoplasmas from rheumatoid synovial fluid. In: Duthie JJR, Alexander WRM, eds. Rheumatic diseases (Pfizer medical monographs). Edinburgh: University Press, 1968:171-81.

4 Schaeverbeke T, Renaudin H, Clerc MC, de Barbeyrac B, Bébéar C, Bannwarth B, Dehais J. Mycoplasmas and arthritis: a systematic culture study of synovial fluids. Arthritis Rheum 1994;37:S315.

5 Barile MF, Yoshida H, Roth H. Rheumatoid arthritis: new findings on the failure to isolate or detect mycoplasmas by multiple cultivation or serologic procedures and review of the literature. Rev Infect Dis 1991;13:571-82.

6 Katseni VL, Gilroy CB, Ryait BK, Ariyoshi K, Bieniasz PD, Weber JN, Taylor-Robinson D. Mycoplasma fermentans in individuals seropositive and seronegative for HIV-1. Lancet 1993;341:271-3.
7 Cimolai N, Malleson P, Thomas E, Middleton PJ. Mycoplasma pneumoniae-associated arthropathy: confirmation of association by deter-

8 Oen K, Fast M, Post B. Epidemiology of juvenile rheumatoid arthritis in Manitoba, Canada, 1975-92: Cycles in incidence. $\mathcal{F}$ Rheumatol 1995;22: $745-50$.

9 Tully JG, Rose DL, Baseman JB, Dallo SF, Lazzell AL, Davis CP. Mycoplasma pneumoniae and Mycoplasma genitalium in synovial fluid isolate. $\mathcal{F}$ Clin Microbiol 1995;33:1851-5.

10 Taylor-Robinson D, Gilroy CB, Horowitz S, Horowitz J. Mycoplasma genitalium in the joints of two patients with arthritis. Eur f Clin Microbiol Infect Dis 1994;13:1066-9. 